\title{
S-1 Combined with Bi-Weekly Docetaxel for Non-Small Cell Lung Cancer Previously Treated with Platinum-Based Chemotherapy: A Phase II Study of North Japan Lung Cancer Group (NJLCG0701)
}

\author{
Osamu Ishimoto ${ }^{1}$, Tomohiro Sakakibara ${ }^{2}$, Makoto Maemondo ${ }^{3}$, Akira Inoue $^{2}$, Nobumichi Matsubara ${ }^{3}$, Naoto \\ Morikawa $^{4}$, Koichi Okudera ${ }^{5}$, Kazuhiro Usui ${ }^{6}$, Toshiro Suzuki ${ }^{7}$, Toshihiro Nukiwa ${ }^{2} \&$ Shunichi Sugawara $^{1}$ \\ ${ }^{1}$ Sendai Kousei Hospital, Sendai, Japan \\ 2 Tohoku University Hospital, Sendai, Japan \\ ${ }^{3}$ Miyagi Cancer Center, Natori, Japan \\ ${ }^{4}$ Tohoku Kousei Nenkin Hospital, Sendai, Japan \\ ${ }^{5}$ Hirosaki Chuo Hospital, Hirosaki, Japan \\ ${ }^{6}$ NTT Medical Center Tokyo, 5-9-22 Higashi-Gotanza, Shinagawa, Tokyo, Japan \\ ${ }^{7}$ Iwate Prefecturel Isawa Hospital, 61 Aza-ryugabanba, Mizusawa, Oshu, Japan \\ Correspondence: Shunichi Sugawara, Department of Pulmonary Medicine, Sendai Kousei Hospital, 4-15 \\ Hirosemachi, Aobaku, Sendai, Miyagi, 980-0873, Japan. Tel: 81-22-222-6181. Fax: 81-22-713-8013. Email: \\ swara357@cat-v.ne.jp
}

Received: January 9, 2012 Accepted: January 29, 2012 Online Published: May 1, 2012

doi:10.5539/cco.v1n1p108 URL: http://dx.doi.org/10.5539/cco.v1n1p108

The research is not financed by any organization.

\begin{abstract}
Objectives: We conducted a phase II study to evaluate the combination of bi-weekly docetaxel and S-1, a novel oral fluorouracil derivative, for patients with previously treated non-small cell lung cancer. Methods: Patients received S-1 on days 1-14 and docetaxel on days 1 and 15 of each 28-day cycle. The primary endpoint was overall response rate (ORR). Results: We enrolled 35 pts from 7 institutions (Feb. 2007-Sep. 2008). Patient characteristics: male/female, 23/12; median age, 64 years; and PS, 0/1 (17/18). The ORR was 26\% (95\% confidence interval, 11-40). The median progression-free survival was 4.1 months and the median overall survival was 16.3 months. Hematologic grade $3 / 4$ toxicity included neutropenia (31\%) and anemia (11\%). Major non-hematologic grade 3 toxicity included diarrhea (17\%). Conclusions: The combination of S-1 and bi-weekly docetaxel is an active regimen with a tolerable toxicity for previously treated NSCLC. Further evaluation of this regimen is warranted.
\end{abstract}

Keywords: chemotherapy, bi-weekly, docetaxel, S-1, phase II trial, non-small cell lung cancer

\section{Introduction}

Fluoropyrimidines are effective anti-cancer drugs against many types of cancer, including non-small cell lung cancer (NSCLC). As a result of a meta-analysis showing that postoperative adjuvant chemotherapy with Uracil-Tegafur (UFT, an oral fluoropyrimidine) improved survival in Japanese patients with completely resected NSCLC (Hamada et al., 2005), UFT is now widely used in Japan in the treatment of this disease. S-1, a novel oral fluoropyrimidine developed as a new anti-cancer drug in Japan in the 1990s. In a phase II study of S-1 in chemotherapy-naïve advanced NSCLC patients, the response rate was $22 \%$ and the median survival time was 10.2 months (Kawahara et al., 2001). Moreover, the combination of S-1 and cisplatin was effective and tolerable in patients with advanced NSCLC, with an overall response rate of 47\%, a median survival time of 11 months, and a one-year survival rate of $45 \%$ (Ichinose et al., 2004). These results indicate that S-1 has good antitumor activity against NSCLC and that it is a good candidate as a non-platinum agent in the treatment of this disease.

On the other hand, docetaxel is also an effective agent for the treatment of NSCLC. According to American Society of Clinical Oncology (ASCO) guidelines, docetaxel is recommended as second-line chemotherapy for 
pre-treated NSCLC patients (Azzoli et al., 2009). However, docetaxel for pre-treated NSCLC showed low response rates (7-11\%) and limited survival benefit (Azzoli et al., 2009). Therefore, more effective regimen as a second line chemotherapy is urgently needed. Its safety and efficacy suggest the combination of daily oral S-1 and bi-weekly docetaxel as an attractive non-platinum option in second-line chemotherapy for previously treated NSCLC patients.

Based on these findings, we conducted a phase II study to evaluate the efficacy and toxicity of this combination as second-line treatment for patients previously treated with platinum-based chemotherapy.

\section{Patients and Methods}

\subsection{Patient Eligibility}

The study population consisted of patients between 20 and 74 years of age with cytologically or histologically confirmed NSCLC with measurable lesions and disease progression after one prior platinum-based chemotherapy regimen. Gefitinib or postoperative adjuvant chemotherapy was not included as prior treatment because it is unlikely to develop drug resistance of our regimen. Other eligibility criteria included an Eastern Cooperative Oncology Group (ECOG) performance status (PS) of 0 or 1 and the ability of the patient to take oral medicine. Laboratory requirements included hemoglobin $>9.0 \mathrm{~g} / \mathrm{dl}$, white blood cell count $>3,000 / \mathrm{mm}^{3}$, neutrophils $>2,000 / \mathrm{mm}^{3}$, platelets $>100,000 / \mathrm{mm}^{3}$, total bilirubin $<1.5 \mathrm{mg} / \mathrm{dl}$, transaminase $<2.5 \mathrm{x}$ the institutional upper limit of the normal value (ULN), serum creatinine $<U L N$, Cockcroft creatinine clearance $>$ $50 \mathrm{ml} / \mathrm{min}$, and $\mathrm{PaO} 2>60 \mathrm{mmHg}$. Patients were ineligible if they had symptomatic brain metastases, pleural, peritoneal, or pericardiac effusion requiring drainage, active double cancer or a severe comorbidity contraindicating chemotherapy, including symptomatic cardiovascular disease, uncontrolled diabetes, pulmonary fibrosis obvious in a chest X-ray, diarrhea, and infectious diseases. Patients who were pregnant, intending to become pregnant, or breast-feeding, patients with a history of drug allergy, patients who had received other fluoropyrimidines or flucytosine, and patients with previous chemotherapy with S-1 or docetaxel were also excluded. The study protocol was approved by the institutional review board of each hospital concerned and written informed consent was obtained from each patient.

\subsection{Drug Administration and Modification}

We had previously tried a combination with biweekly docetaxel and S-1 for previously treated NSCLC as a phase I study. Briefly, the dose of docetaxel was escalated from $20 \mathrm{mg} / \mathrm{m}^{2}$ to $30 \mathrm{mg} / \mathrm{m}^{2}$ by $5 \mathrm{mg} / \mathrm{m}^{2}$ based on toxicity during the first cycle of chemotherapy. The dose of S-1 was fixed to $80 \mathrm{mg} / \mathrm{m}^{2} /$ day for 2 weeks. The maximum tolerated dose (MTD) was defined as the dose level at which more than two of three patients or more than two of six patients experienced dose limiting toxicities. The dose recommended for the following phase II study was defined as the dose preceding the MTD. This phase I study revealed the recommended dose of docetaxel was $25 \mathrm{mg} / \mathrm{m}^{2}$. Based on these data, docetaxel $\left(25 \mathrm{mg} / \mathrm{m}^{2}\right)$ was administered intravenously on days 1 and 15 and oral S-1 twice daily after meals for 2 weeks followed by a 2-week rest during each 28-day cycle. The dose of S-1 was modified according to body surface area (BSA) and was approximately $80 \mathrm{mg} / \mathrm{m}^{2} /$ day: $\mathrm{BSA}<1.25 \mathrm{~m}^{2}, 80 \mathrm{mg} /$ day; $1.25 \leq \mathrm{BSA}<1.5 \mathrm{~m}^{2}, 100 \mathrm{mg} /$ day; $\mathrm{BSA} \geq 1.5 \mathrm{~m}^{2}, 120 \mathrm{mg} /$ day. Patients were allowed to receive premedication, including antiemetics and streroids, prior to administration of anticancer agents, but prophylactic granulocyte colony-stimulating factor or prophylactic antibiotic support was not allowed.

Toxicities were assessed according to the Common Terminology Criteria for Adverse Events version 3.0. Docetaxel on day 15 was administered only if the leukocyte count was over $2,000 / \mathrm{mm}^{3}$, the neutrophil count over $1,000 / \mathrm{mm}^{3}$, and the platelet count over $75,000 / \mathrm{mm}^{3}$. S-1 was stopped if the leukocyte count was < $2,000 / \mathrm{mm}^{3}$, the neutrophil count $<1,000 / \mathrm{mm}^{3}$, or the platelet count $<50,000 / \mathrm{mm}^{3}$. Administration of both S-1 and docetaxel was stopped if serum creatinine was over the institutional upper limit of the normal value or transaminase was over $2.5 \times \mathrm{ULN}$, total bilirubin $>1.5 \mathrm{mg} / \mathrm{dl}$, or if the patient experienced any non-hematological toxicity $\geq$ grade 3 , except for nausea, alopecia, anorexia, or fatigue.

Dose reduction in subsequent cycles was permitted according to the schedule below if any of the following toxicities occurred: S-1 was reduced by $20-30 \mathrm{mg} /$ body/day if a leukocyte count of lower than $1,000 / \mathrm{mm}^{2}$, grade 4 neutropenia lasting $\geq 4$ days, febrile neutropenia, or grade 3 or more major non-hematological toxic effects excluding anorexia, nausea, or fatigue was observed. If such toxicities persisted after dose reduction, docetaxel dose was reduced to $20 \mathrm{mg} / \mathrm{m}^{2}$ in the next cycle.

It was intended that the treatment be continued unless disease progression or unacceptable toxicity occurred, the patient refused further treatment, or the physician decided to discontinue the treatment. Treatment given after completion of the study was not restricted by the study protocol. 


\subsection{Treatment Assessment}

Baseline assessment included a physical examination, complete blood cell counts (CBC), hepatic and renal functions tests, urinalysis, 12-lead electrocardiogram, and chest X-ray. Measurements of visible and palpable tumors were performed at baseline by chest X-ray, computed tomography (CT), or magnetic resonance imaging. During the study, the medical history and results of physical examination, weight, vital signs, ECOG performance status, $\mathrm{CBC}$, and blood chemistry were monitored every 2 weeks. Radiographic evaluation by CT by extramural review was performed to assess response to treatment. Unidimensional measurements were undertaken using the RECIST criteria (Therasse et al., 2000). For the confirmation of response according to the RECIST criteria, a response $>4$ weeks duration was needed for a complete response (CR) or partial response (PR) and $>6$ weeks from the initiation of chemotherapy was needed to determine the disease as stable.

\subsection{Study Design and Statistical Analysis of Phase II Trial}

The primary objective of the trial was to determine response rate, defined as the proportion of the patients who attained CR or PR. Simon's two-stage optimal design was used to determine the sample size (Simon, 1989). Previous phase III trials reported that the response rates of tri-weekly docetaxel in pretreated NSCLC were between 2.7\% and 12.6\% (Camps et al, 2006; Gridelli et al, 2004; Schuette et al, 2005). On the other hand, a phase II study of bi-weekly docetaxel showed $20 \%$ of response rate as a second line chemotherapy (Vázquez et $\mathrm{al}, 2004)$. Assuming that a response rate of $20 \%$ in eligible patients would indicate potential usefulness while a rate of $5 \%$ would be the lower limit of interest, along with alpha and beta values of 0.05 and 0.10 , respectively, the estimated accrual was 34 pts. The secondary endpoints were toxicity, progression-free survival (PFS) and overall survival (OS). Survival was defined as the interval between the date of registration and date of death or last follow-up visit. Survival distribution was estimated by the Kaplan-Meier method.

\section{Results}

\subsection{Patient Characteristics}

Between February 2007 and September 2008, 35 NSCLC patients were enrolled from 7 participating institutions. Patient characteristics are summarized in Table 1. Seventeen patients had a PS of 1 and 7 patients $(20 \%)$ were elderly ( $>70$ years old). The predominant histology was adenocarcinoma (66\%). Eighteen patients $(51 \%)$ were stage III and 12 patients (34\%) were stage IV, and $5(14 \%)$ had postoperative recurrence with distant metastasis when first diagnosed. Best overall response rate to first-line platinum-based chemotherapy was $71 \%$. Ten of the stage III patients received thoracic radiotherapy combined with platinum-based chemotherapy, and 8 of them obtained PR. Epidermal growth factor receptor (EGFR) status was examined in 16 patients, including 3 (19\%) patients who harbored EGFR-tyrosine kinase inhibitor (TKI)-sensitive mutations. Gefitinib, an EGFR-TKI, was used in 5 patients as prior treatment, and 2 of these patients were responsive to gefitinib. 
Table 1. Patient characteristics

\begin{tabular}{|c|c|}
\hline & No. of Patients \\
\hline Enrolled patients & 35 \\
\hline \multicolumn{2}{|l|}{ Sex } \\
\hline Male/Female & $22 / 13$ \\
\hline \multicolumn{2}{|l|}{ Age (yrs) } \\
\hline Median (range) & $64(43-74)$ \\
\hline PS* $0 / 1$ & $18 / 17$ \\
\hline
\end{tabular}

Histology

Adenocarcinoma $\quad 23$

Squamous cell carcinoma $\quad 8$

Large cell carcinoma $\quad 1$

Adenosquamous cell carcinoma $\quad 1$

Other 2

\begin{tabular}{ll}
\hline Disease stage at first-line therapy & 5 \\
IIIA $^{\#}$ & 13 \\
IIIB $^{\#}$ & 12 \\
IV & 5 \\
Postoperative relapse** & \\
\hline
\end{tabular}

\begin{tabular}{ll}
\hline Best response to platinum & 1 \\
CR & 24 \\
PR & 9 \\
SD & 1 \\
PD &
\end{tabular}

* Eastern Cooperative Oncology Group performance status

\# 10 patients of stage III disease received platinum-based chemoradiotherapy.

** No patients received postoperative adjuvant chemotherapy.

\subsection{Tumor Response and Survival}

Thirty-five patients received a total of 101 cycles of the protocol treatment, and the median number of cycles was 3 (range, 1-10). Ninety-eight percent of the planned dose of S-1 and $90 \%$ of the planned dose of docetaxel was administered. No patient required a dose reduction because of adverse effects.

Two patients could not be evaluated for response (Table 2). The overall response rate was $25.7 \%$ (95\% confidence interval [CI], 11.2\%-40.2\%). Stable disease was observed in 14 patients (40\%) and PD in 10 patients (29\%). In adenocarcinoma patients, 30\% (7/23) experienced PR, whereas $25 \%(2 / 8)$ of squamous cell carcinoma patients obtained PR.

Table 2. Response

\begin{tabular}{ll}
\hline & No. of Patients \\
\hline Complete response & 0 \\
Partial response & 9 \\
Stable disease & 14 \\
Progressive disease & 10 \\
Not evaluable & 2 \\
\hline
\end{tabular}

Overall response rate $25.7 \%(95 \% \mathrm{CI}, 11.2-40.2 \%)$

Disease control rate $65.7 \%(95 \% \mathrm{CI}, 50.0-81.4 \%)$ 
Survival analysis was performed in December 2009. Median follow-up time for the living patients was 18.5 months (range, 13.9-32.3 months). Median PFS was 4.1 months (Figure 1A). The median survival time (MST) of all patients was 16.2 months, and the 1 -year survival rate was $61 \%$ (Figure 1B). In adenocarcinoma patients, the median PFS was 3. 8 months (range, 1.0-15.1 months) and MST was 14.7 months (range, 1.1-30.9 months). In squamous cell carcinoma patients, the median PFS was 4.3 months (range, 2.0-11.4 months) and MST was 16.7 months (range, 6.9-22.7 months)

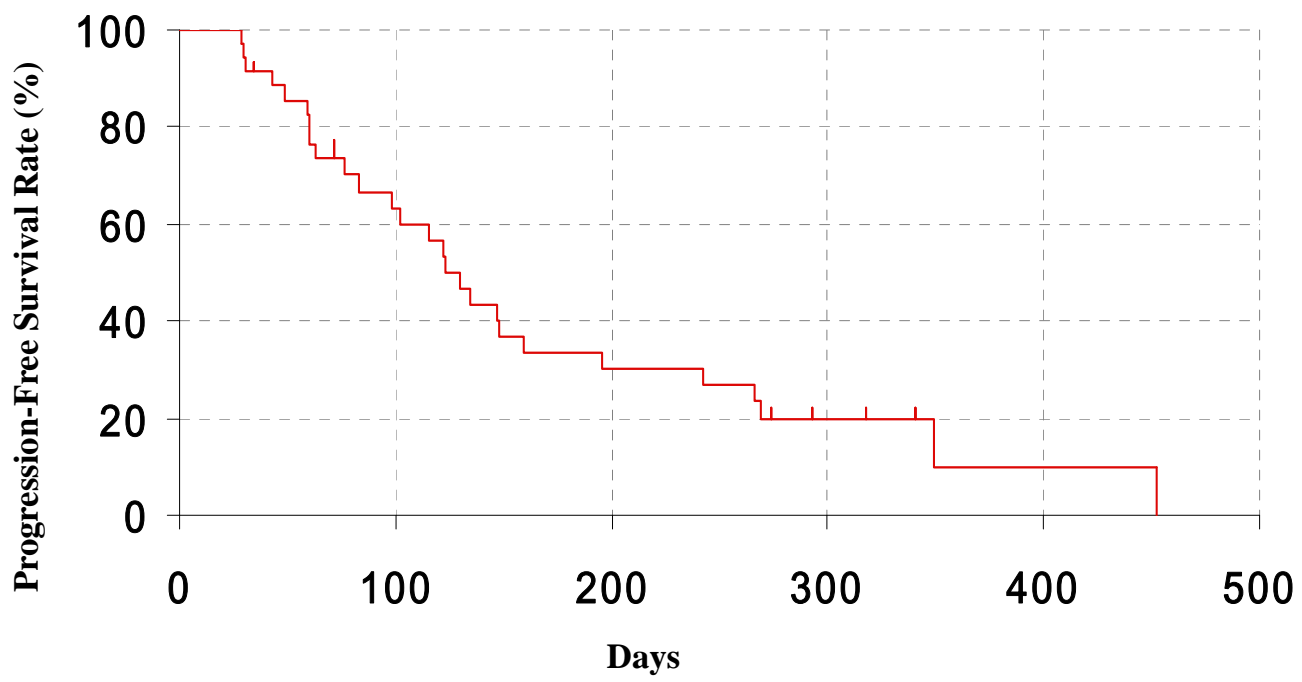

Figure 1A. Progression free survival by Kaplan-Meier method. Median progression free survival was 4.1 months

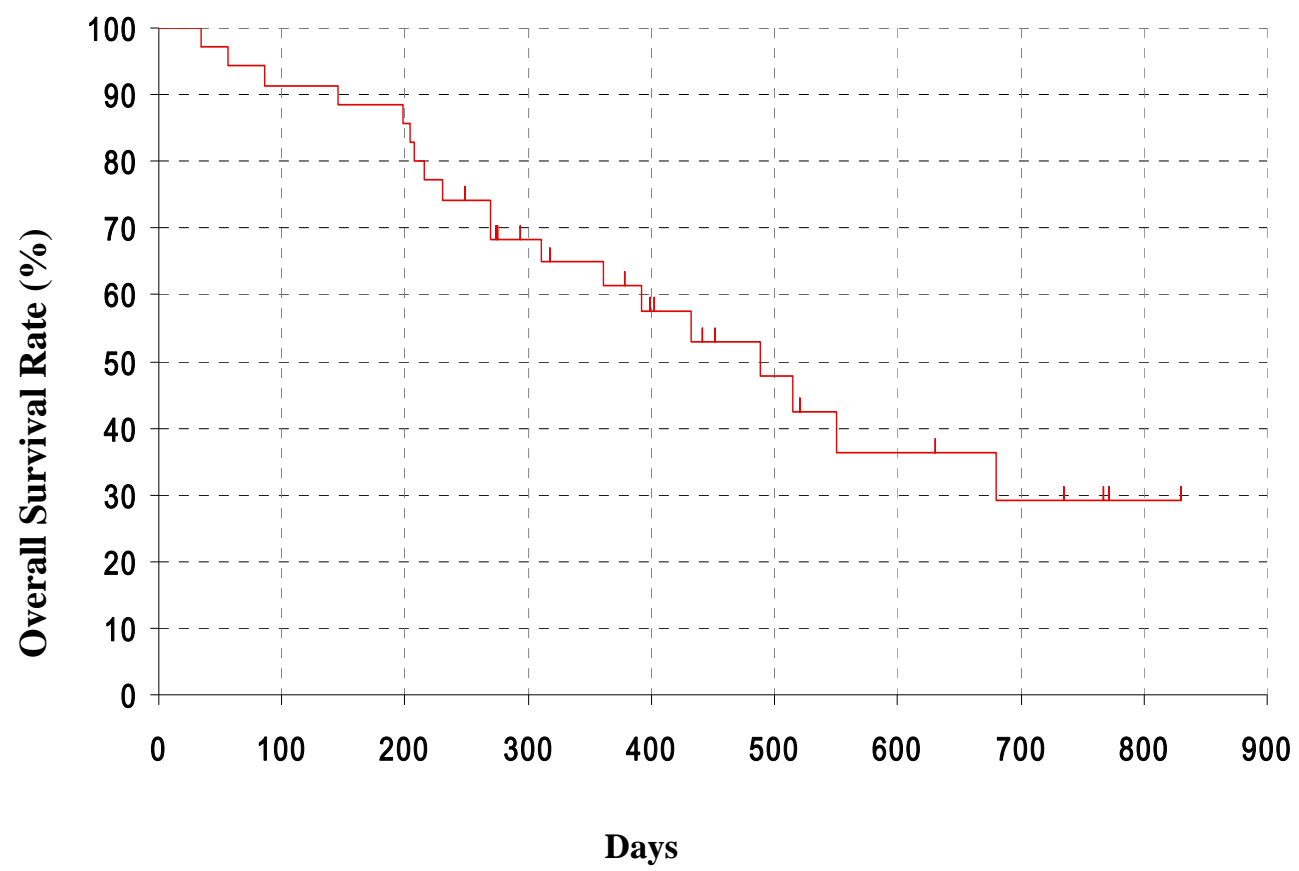

Figure 1B. Overall survival by Kaplan-Meier method. Median survival time was 16.2 months. 1-year survival rate was $61 \%$

Twenty-three patients $(66 \%)$ received post-study treatment. Sixteen patients were treated with other cytotoxic chemotherapy and 9 patients were treated with EGFR-TKIs.

\subsection{Toxicity}

All patients were assessable for toxicity. Of the 8 patients who received only one cycle, treatment was terminated 
in 3 patients due to rapid disease progression, while treatment was discontinued in the remaining 5 patients due to toxicity. Hematologic grade $3 / 4$ toxicity included neutropenia $(31 \%)$ and anemia $(11 \%)$. No febrile neutropenia was observed (Table 3). Major non-hematologic grade 3 toxicities included diarrhea (17\%) and infection $(8.6 \%)$. No grade 4 non-hematologic toxicity was observed. Other toxicities were mostly mild to moderate and tolerable. However, there was one possible treatment-related death due to pneumonitis after the first cycle of chemotherapy (Table 4).

Table 3. Hematological Toxicity (all cycles)

\begin{tabular}{llllll}
\hline NCI-CTC grade & $\mathbf{1}$ & $\mathbf{2}$ & $\mathbf{3}$ & $\mathbf{4}$ & Grade $\mathbf{3 / 4}$ \\
\hline Leukopenia & 1 & 8 & 5 & 1 & $6(17 \%)$ \\
Neutropenia & 2 & 2 & 8 & 3 & $11(31 \%)$ \\
Anemia & 18 & 7 & 4 & 0 & $4(11 \%)$ \\
Thrombocytopenia & 4 & 2 & 0 & 0 & 0 \\
Febrile neutropenia & - & - & 0 & 0 & 0
\end{tabular}

Toxicities were graded according to National Cancer Institute toxicity criteria (NCI-CTC) during all cycles of chemotherapy

Table 4. Non-hematological Toxicity (all cycles)

\begin{tabular}{|c|c|c|c|c|c|c|}
\hline NCI-CTC grade & 1 & 2 & 3 & 4 & 5 & Grade 3/4 \\
\hline Anorexia & 8 & 5 & 2 & & & $2(5.7 \%)$ \\
\hline Nausea/Vomiting & 8 & 4 & 1 & & & $1(2.9 \%)$ \\
\hline Diarrhea & 2 & 1 & 6 & & & $6(17 \%)$ \\
\hline Hemorrhage, Lower GI & 1 & & & & & \\
\hline Bilirubin & 4 & 1 & & & & \\
\hline ALT/AST & 10 & 3 & 2 & & & $2(5.7 \%)$ \\
\hline Hypoalbuminemia & 12 & 3 & & & & \\
\hline ALP & 2 & & & & & \\
\hline $\mathrm{Na} / \mathrm{K}$ & 13 & & & & & \\
\hline Hypocalcemia/Creatinine & 2 & & & & & \\
\hline Mucositis & 5 & 3 & 1 & & & $1(2.9 \%)$ \\
\hline Pneumonitis & & & & & $1 *$ & $1(2.9 \%)$ \\
\hline Pneumothorax & & & 1 & & & $1(2.9 \%)$ \\
\hline Dyspnea & & & 1 & & & $1(2.9 \%)$ \\
\hline Rash/Hyperpigmentation & 5 & 2 & 2 & & & $2(5.7 \%)$ \\
\hline Nail changes & & 1 & & & & \\
\hline Neuropathy-sensory & 1 & 2 & & & & \\
\hline Alopecia & 6 & 1 & & & & \\
\hline Fever & 2 & 2 & & & & \\
\hline Infection & & 1 & 3 & & & $3(8.6 \%)$ \\
\hline Insomnia & 1 & & & & & \\
\hline
\end{tabular}

Toxicities were graded according to National Cancer Institute toxicity criteria (NCI-CTC) during all cycles of chemotherapy.

*Died due to pneumonitis after first cycle of chemotherapy 


\section{Discussion}

In this study, we performed a phase II trial of S-1 in combination with bi-weekly docetaxel in patients with pre-treated NSCLC. The objective response rate was $26 \%$ (95\% CI, 11-40), which met the primary endpoint of the study. In addition, this regimen had a mild toxicity profile.

\subsection{Non-Platinum Regimen}

At present, combination chemotherapy with platinum and third-generation anti-tumor drugs is considered the standard approach to the treatment of chemotherapy-naïve advanced/recurrent NSCLC patients. However, several randomized comparative studies investigating non-platinum regimens including third-generation anti-tumor drugs found they resulted in a comparable effect and less toxicity than platinum-based regimens for NSCLC (Georgoulias et al, 2001; Kosmidis et al, 2002; Smit et al, 2003; Georgoulias et al, 2005; Pujol et al, 2005). Therefore, the development of novel non-platinum regimens for NSCLC is attractive. In addition, second-line chemotherapy is also important in treating patients who are refractory to platinum doublets or in whom such regimens are intolerable. According to the 2009 ASCO guidelines, docetaxel, pemetrexed or EGFR-TKIs are recommended as second-line therapy after failure of platinum-based chemotherapy. This recommendation is based on several studies that showed an overall benefit for these drugs (Azzoli et al, 2009).

\subsection{Treatment Schedule}

Several studies comparing different dosages or schedules of administration of docetaxel showed that weekly administration of docetaxel had similar advantages to tri-weekly administration, although hematologic toxicities were significantly more severe in the tri-weekly schedule (Camps et al, 2006; Gridelli et al, 2004; Schuette et al, 2005). In providing salvage chemotherapy, pursuing its efficacy is needed, but maintaining quality of life is also required. In the present study, the addition of S-1 was expected to improve its efficacy, and docetaxel was split bi-weekly to improve its toxicity. There are two reasons why we selected a bi-weekly schedule of docetaxel. First, the development of a weekly regimen of docetaxel was terminated due to an unexpectedly high rate of interstitial pneumonitis in Japan [unpublished data]. Therefore, we considered that weekly docetaxel combined with S-1 might incur a significant risk of lung toxicity. Second, an earlier study found that bi-weekly docetaxel was effective for previously treated NSCLC patients, yielding a $20 \%$ response rate, a 2.8 -month PFS and 4.0-month OS with tolerable toxicity (Vázquez et al, 2004). Therefore, we selected a bi-weekly schedule for this study.

\subsection{Combination of S-1 and Docetaxel}

S-1 consists of a 1:0.4:1 molar ratio of tegafur, 5-chloro-2, 4-dihydroxypyridine (CDHP) and potassium oxonate (OXO) (Shirasaka et al., 1996). Tegafur is a prodrug of 5-fluorouracil (5-FU), whereas CDHP is an inhibiter of dihydropyrimidine dehydrogenase, the enzyme responsible for degradation of 5-FU. Compared with tegafur alone, tegafur plus CDHP increased 5-FU concentration in serum and tumor tissue by inhibiting 5-FU degradation (Shirasaka et al., 1996). One of the dose limiting toxicities of 5-FU is gastrointestinal toxicity, which is associated with its phosphoribosylation by the enzyme orotate phosphoribosyl transferase (OPRT). As S-1 includes OXO, an inhibiter of OPRT, a palliative effect may be expected on any gastrointestinal toxicities arising from selective concentration of tegafur in gastrointestinal tissues after oral administration (Shirasaka et al., 1993). The combination of S-1 and docetaxel has been intensively investigated in gastric cancer. S-1 monotherapy for gastric cancer was ineffective when thymidylate synthase (TS) was highly expressed in tumors. However, a synergistic effect was observed when 5-FU was combined with docetaxel, which decreased expression of TS and dihydropyrimidine dehydrogenase (DPD). In vitro and in vivo analyses showed that docetaxel modulated these 5-FU metabolic enzymes (Wada et al, 2006; Takahashi et al, 2005). In addition, plasma pharmacokinetic analysis in phase I studies of S-1 plus bi-weekly docetaxel in patients with gastric cancer demonstrated that administration of S-1 had no effect on plasma concentrations of docetaxel (Takahashi et al, 2006). S-1 monotherapy as second-line chemotherapy for NSCLC showed a $12.5 \%$ overall response rate and 2.5 months median PFS (Totani et al, 2009). Yanagihara et al. (2010) and Atagi et al. (2008) administered S-1 plus tri-weekly docetaxel $\left(40 \mathrm{mg} / \mathrm{m}^{2}\right)$ in patients with previously treated NSCLC and found that the overall response rate was $18.4 \%$ and $24.1 \%$, respectively. The response rate of our regimen $(26 \% ; 95 \%$ confidence interval, $11-40)$ was comparable with these results, and median PFS was almost identical at 4.4 months, 3.9 months and 4.1 months, respectively. In addition, Oki et al. (2011) reported a study of S-1 combined with bi-weekly docetaxel $\left(35 \mathrm{mg} / \mathrm{m}^{2}\right)$, which was the same treatment schedule as this study. Although they administered higher dose of docetaxel, their regimen showed the similar response rate $(16.3 \%)$ with higher toxicity. Taken together with the results of these earlier reports, our data suggest that addition of docetaxel improves the efficacy of S-1, and that a bi-weekly schedule of docetaxel in combination with S-1 is similarly active to a tri-weekly schedule. 


\subsection{Toxicity}

Only mild hematological toxicity was observed in this study. Tri-weekly docetaxel plus S-1 induced grade 3/4 neutropenia in 50\% (Yanagihara et al., 2010) and 34.5\% (Atagi et al., 2008) of patients in earlier studies, compared with in only $31 \%$ of our patients.

Non-hematological toxicities in this study were also mild. However, we should mention that $2.9 \%$ and $17 \%$ of our patients experienced grade 5 pneumonitis and grade 3 diarrhea, respectively. Although there are no data showing that bi-weekly docetaxel incurs greater risk of pneumonitis than tri-weekly docetaxel, weekly docetaxel induced pneumonitis frequently in Japan [unpublished data], so potential lung toxicity must be taken into consideration. It is possible that S-1 is a potent inducer of severe diarrhea, as grade 3 or 4 diarrhea was reported in $8.5 \%$ of NSCLC patients on monotherapy with S-1 (Kawahara et al., 2001). One treatment related death was observed both in this study and that of Atagi et al. (2008).

Although the toxicity profile needs to be assessed in further study, the current results suggest that S-1 plus bi-weekly docetaxel would be useful as an outpatient-based chemotherapy regimen.

\subsection{Histology and S-1}

Thymidylate synthase, the main target of pemetrexed and fluoropyrimidine, is differentially expressed depending on histological type of lung cancer. For example, TS is more highly expressed in squamous cell lung cancer than in adenocarcinoma (Otake et al., 1999). Differential expression of TS depending on histological type of lung cancer is believed to affect clinical response to pemetrexed. Non-squamous patients treated with pemetrexed had longer survival than those treated with gemcitabine or docetaxel (Scagliotti et al., 2009). However, in the present study, S-1 in combination with bi-weekly docetaxel showed similar activity to that in adenocarcinoma (response rate, 30\%; median PFS, 3.8 months) and squamous cell carcinoma (response rate, 25\%; median PFS, 4.3 months). In addition, a pooled analysis of S-1 trials found that there was no difference in response to S-1 in combination with cisplatin depending on histological type (Yamamoto et al., 2010). Lung cancer showed relatively high expression of DPD, which causes greater degradation of 5-FU than other cancers (Fukui et al., 2008). Therefore, being a DPD-inhibitory fluoropyrimidine, S-1 might prevent overexpression of TS, which would make it a good candidate for the treatment of squamous cell carcinoma. Although larger scale study is required to confirm this, addition of docetaxel to S-1 appears to be a promising combination chemotherapy regimen in the treatment of lung cancer, irrespective of histological type.

\subsection{Limitations}

Overall survival time was prolonged, in spite of a relatively short PFS. However, several limitations must be taken into consideration. $14 \%$ of our patients were stage IIIA at diagnosis and had previously been treated with chemoradiotherapy. Seventy-one percent (25/35) of our patients were responsive (CR or PR) to first-line therapy, and $66 \%$ of the patients received post-study treatment including EGFR-TKIs. Lastly, the relatively small number of patients $(n=35)$, relatively young patients $(80 \%$ of patients were less than 70 years of age) with good performance status (half patients showed PS 0) were enrolled in this study. Considering the sample was not fully homogeneous, it is possible that our regimen did not actually contribute to the observed prolonged overall survival time, and this needs to be investigated in further randomized study.

\subsection{Conclusions}

In conclusion, we found that bi-weekly docetaxel (day 1,15) combined with oral S-1 (day 1-14) was an active regimen with a mild toxicity profile for previously treated NSCLC. The convenience and safety of this regimen may be appropriate for outpatients. To evaluate its superiority in terms of risk-benefit balance, further evaluation of this regimen is required in comparison with administration of docetaxel alone or pemetrexed.

\section{Acknowledgements}

We thank Associate Professor Jeremy Williams of Tokyo Dental College Oral Health Science Center for his critical review of this manuscript.

\section{References}

Atagi, S., Kawahara, M., Kusunoki, Y., Takada, M., Kawaguchi, T., Okishio, K., ... Komatsu, H. (2008). Phase I/II study of docetaxel and S-1 in patients with previously treated non-small cell lung cancer. $J$ Thorac Oncol, 3, 1012-1017. http://dx.doi.org/10.1097/JTO.0b013e318183f8ed

Azzoli, C. G., Baker, S. Jr., Temin, S., Pao, W., Aliff, T., Brahmer, J., ... Giaccone, G. (2009). American Society of Clinical Oncology. American Society of Clinical Oncology Clinical Practice Guideline update on chemotherapy for stage IV non-small-cell lung cancer. $J$ Clin Oncol, 27, 6251-6266. 
http://dx.doi.org/10.1200/JCO.2009.23.5622

Camps, C., Massuti, B., Jiménez, A., Maestu, I., Gómez, R. G., Isla, D., ... Felip, E. (2006). Spanish Lung Cancer Group. Randomized phase III study of 3-weekly versus weekly docetaxel in pretreated advanced non-small-cell lung cancer: a Spanish Lung Cancer Group trial. Ann Oncol, 17, 467-472. http://dx.doi.org/10.1093/annonc/mdj115

Fukui, Y., Oka, T., Nagayama, S., Danenberg, P. V., Danenberg, K. D., \& Fukushima, M. (2008). Thymidylate synthase, dihydropyrimidine dehydrogenase, orotate phosphoribosyltransferase mRNA and protein expression levels in solid tumors in large scale population analysis. Int $J$ Mol Med, 22, 709-716. http://dx.doi.org/10.3892/ijmm_00000076

Georgoulias, V., Papadakis, E., Alexopoulos, A., Tsiafaki, X., Rapti, A., Veslemes, M., Palamidas, P., \& Vlachonikolis, I. (2001). Greek Oncology Cooperative Group (GOCG) for Lung Cancer. Platinum-based and non-platinum-based chemotherapy in advanced non-small-cell lung cancer: a randomised multicentre trial. Lancet, 357, 1478-1484. http://dx.doi.org/10.1016/S0140-6736(00)04644-4

Georgoulias, V., Ardavanis, A., Tsiafaki, X., Agelidou, A., Mixalopoulou, P., Anagnostopoulou, O., ... Chatzidaki, D. (2005). Vinorelbine plus cisplatin versus docetaxel plus gemcitabine in advanced non-small-cell lung cancer: a phase III randomized trial. J Clin Oncol, 23, 2937-2945. http://dx.doi.org/10.1200/JCO.2005.04.016

Gridelli, C., Gallo, C., Di Maio, M., Barletta, E., Illiano, A., Maione, P., ... Perrone, F. (2004). A randomised clinical trial of two docetaxel regimens (weekly vs 3 week) in the second-line treatment of non-small-cell lung cancer. The DISTAL 01 study. Br J Cancer, 91, 1996-2004. http://dx.doi.org/10.1038/sj.bjc.6602241

Hamada, C., Tanaka, F., Ohta, M., Fujimura, S., Kodama, K., Imaizumi, M., \& Wada, H. (2005). Meta-analysis of postoperative adjuvant chemotherapy with tegafur-uracil in non-small-cell lung cancer. J Clin Oncol, 23, 4999-5006. http://dx.doi.org/10.1200/JCO.2005.09.017

Ichinose, Y., Yoshimori, K., Sakai, H., Nakai, Y., Sugiura, T., Kawahara, M., \& Niitani, H. (2004). S-1 plus cisplatin combination chemotherapy in patients with advanced non-small cell lung cancer: a multi-institutional phase II trial. Clin Cancer Res, 10, 7860-7864. http://dx.doi.org/10.1158/1078-0432.CCR-04-1200

Kawahara, M., Furuse, K., Segawa, Y., Yoshimori, K., Matsui, K., Kudoh, S., ... Niitani, H. (2001). S-1 Cooperative Study Group (Lung Cancer Working Group. Phase II study of S-1, a novel oral fluorouracil, in advanced non-small-cell lung cancer. Br J Cancer, 85, 939-943. http://dx.doi.org/10.1054/bjoc.2001.2031.

Kosmidis, P., Mylonakis, N., Nicolaides, C., Kalophonos, C., Samantas, E., Boukovinas, J., ... Dimopoulos, M. (2002). Paclitaxel plus carboplatin versus gemcitabine plus paclitaxel in advanced non-small-cell lung cancer: a phase III randomized trial. J Clin Oncol, 20, 3578-3585. http://dx.doi.org/10.1200/JCO.2002.12.112

Oki, Y., Hirose, T., Yamaoka, T., Kusumoto, S., Shirai, T., Sugiyama, T., ... Adachi, M. (2011). Phase II study of S1 and biweekly docetaxel in previously treated NSCLC. Cancer Chemother Pharmacol, 67(4), 791-797. http://dx.doi.org/10.1007/s00280-010-1382-1

Otake, Y., Tanaka, F., Yanagihara, K., Hitomi, S., Okabe, H., Fukushima, M., \& Wada, H. (1999). Expression of thymidylate synthase in human non-small cell lung cancer. Jpn J Cancer Res, 90, 1248-1253.

Pujol, J. L., Breton, J. L., Gervais, R., Rebattu, P., Depierre, A., Morère, J. F., ... Clary, C. (2005). Gemcitabine-docetaxel versus cisplatin-vinorelbine in advanced or metastatic non-small-cell lung cancer: a phase III study addressing the case for cisplatin. Ann Oncol, 16, 602-610. http://dx.doi.org/10.1093/annonc/ $\operatorname{mdi} 126$

Scagliotti, G., Hanna, N., Fossella, F., Sugarman, K., Blatter, J., Peterson, P., ... Shepherd, F. A. (2009). The differential efficacy of pemetrexed according to NSCLC histology: a review of two Phase III studies. Oncologist, 14, 253-263. http://dx.doi.org/10.1634/theoncologist.2008-0232

Schuette, W., Nagel, S., Blankenburg, T., Lautenschlaeger, C., Hans, K., Schmidt, E. W., ... Serke, M. (2005). Phase III study of second-line chemotherapy for advanced non-small-cell lung cancer with weekly compared with 3-weekly docetaxel. J Clin Oncol, 23, 8389-8395. http://dx.doi.org/10.1200/JCO.2005.02. 3739

Shirasaka, T., Shimamato, Y., Ohshimo, H., Yamaguchi, M., Kato, T., Yonekura, K., \& Fukushima, M. (1996). Development of a novel form of an oral 5-fluorouracil derivative (S-1) directed to the potentiation of the 
tumor selective cytotoxicity of 5-fluorouracil by two biochemical modulators. Anticancer Drugs, 7, 548-557.

Shirasaka, T., Shimamoto, Y., \& Fukushima, M. (1993). Inhibition by oxonic acid of gastrointestinal toxicity of 5-fluorouracil without loss of its antitumor activity in rats. Cancer Res, 53, 4004-4009.

Simon, R. (1989). Optimal two-stage designs for phase II clinical trials. Control Clin Trials, 10 , 1-10. http://dx.doi.org/10.1016/0197-2456(89)90015-9

Smit, E. F., van Meerbeeck, J. P., Lianes, P., Debruyne, C., Legrand, C., Schramel, F., ... Giaccone, G.; European Organization for Research and Treatment of Cancer Lung Cancer Group. (2003). Three-arm randomized study of two cisplatin-based regimens and paclitaxel plus gemcitabine in advanced non-small-cell lung cancer: a phase III trial of the European Organization for Research and Treatment of Cancer Lung Cancer Group--EORTC 08975. J Clin Oncol, 21, 3909-3917. http://dx.doi.org/10.1200/JCO.2003.03.195

Takahashi, I., Emi, Y., Kakeji, Y., Uchida, J., Fukushima, M., \& Maehara, Y. (2005). Increased antitumor activity in combined treatment TS-1 and docetaxel. A preclinical study using gastric cancer xenografts. Oncology, 68, 130-137. http://dx.doi.org/10.1159/000086767

Takahashi, I., Emi, Y., Kakeji, Y., Tokunaga, E., Ushiro, S., Oki, E., ... Maehara, Y. (2006). Phase I study of S-1 and biweekly docetaxel combination chemotherapy for advanced and recurrent gastric cancer. Oncol Rep, $15,849-854$.

Therasse, P., Arbuck, S. G., Eisenhauer, E. A., Wanders, J., Kaplan, R. S., Rubinstein, L., ... Gwyther, S. G. (2000). New guidelines to evaluate the response to treatment in solid tumors (RECIST Guidelines). $J$ Natl Cancer Inst, 92, 205-16. http://dx.doi.org/10.1093/jnci/92.3.205

Totani, Y., Saito, Y., Hayashi, M., Tada, T., Kohashi, Y., Mieno, Y., ... Sakakibara, H. (2009). A phase II study of S-1 monotherapy as second-line treatment for advanced non-small cell lung cancer. Cancer Chemother Pharmacol, 64, 1181-1185. http://dx.doi.org/10.1007/s00280-009-0981-1

Yamamoto, N., Yamanaka, T., Ichinose, Y., Kubota, K., Sakai, H., Gemma, A., ... Niitani, H. (2010). Pooled analysis of S-1 trials in non-small cell lung cancer according to histological type. Anticancer Res, 30, 2985-2990.

Yanagihara, K., Yoshimura, K., Niimi, M., Yasuda, H., Sasaki, T., Nishimura, T., ... Fukushima, M. (2010). Phase II study of S-1 and docetaxel for previously treated patients with locally advanced or metastatic non-small cell lung cancer. Cancer Chemother Pharmacol, 64(5), 913-8. http://dx.doi.org/10.1007/s00280009-1239-7

Vázquez, S., Grande, C., Amenedo, M., Fírvida, J. L., Lázaro, M., Alonso, G., Curiel, T., ... Ramos, M. (2004). Biweekly docetaxel as second-line chemotherapy of patients with advanced non-small cell lung cancer: a phase II study of the Galician Lung Cancer Group (GGCP 006-00). Anticancer Drugs, 15, 489-494. http://dx.doi.org/10.1097/01.cad.0000127333.06439.0e.

Wada, Y., Yoshida, K., Suzuki, T., Mizuiri, H., Konishi, K., Ukon, K., ... Fukushima, M. (2006). Synergistic effects of docetaxel and S-1 by modulating the expression of metabolic enzymes of 5-fluorouracil in human gastric cancer cell lines. Int J Cancer, 119, 783-791. http://dx.doi.org/10.1002/ijc.21879 